\title{
Common Weights in DEA Models with Reduced Singular Value Decomposition
}

\author{
Hassan Naseri ${ }^{1}$, S.Esmaeil Najafi ${ }^{2}$,Abbas Saghaei ${ }^{3}$ \\ ${ }^{1,2,3}$ Department of Industrial Engineering, Engineering Faculty, Science \& Research \\ Branch, Islamic Azad University, Tehran, Iran \\ Tel: ${ }^{1} 0098-21-81806457,{ }^{2,3} 0098-21-44865154$ \\ E-mail:1hassan.naseri@gmail.com,2najafi1515@yahoo.com,3 \\ abbas.saghaei@gmail.com
}

\begin{abstract}
In DEA framework there are many techniques for finding a common set of efficient weights depend on inputs and outputs values in a set of peer DecisionMaking Units (DMUs). In a lot of papers, has been discussed multiple criteria decision-making techniques and multiple objective-decision criteria for modeling. We know the objective function of a common set of weights is defined like an individual efficiency of one DMU with a basic difference: "trying to maximize the efficiency of all DMUs simultaneously, with unchanged restrictions". An ideal solution for a common set of weights can be the closest set to the derived individual solution of each DMU. Now one question can be: "are the closest set and minimized set, which is found in most of the techniques, are different?" The answer can be: "They are different when the variance between the generated weights of a specific input (output) from n DMUs is big". In this case, we will apply Singular Value Decomposition (SVD) such that, first, the degree of importance weights for each input (output) will be defined and found, then, the Common Set of Weights (CSW) will be found by the closest set to these weights. The degree of importance values will affect the CSW of each DMU directly.
\end{abstract}

Keywords: Data Envelopment Analysis, Multiple objective methods in optimization, Singular value decomposition, CCR, Common Set of Weights

\section{1-Introduction}

Data Envelopment Analysis (DEA), which is a non-parametric method for measuring the efficiency of a set of peer DMUs (such as firms or public sectors), was first introduced into the Operations Research (OR) literature by Charnes, Cooper, Rhodes (CCR, 1978). Today DEA is one of the most powerful tool used for efficiency evaluation, although as Ray Subhash, (2004) has pointed, some economists debate the three main components of DEA: (i) Because DEA is a nonparametric method, no production, cost, or profit function is estimated from the data. This precludes evaluation of marginal products, partial elasticities, marginal costs, or elasticities of substitution from a fitted model. As a result, one cannot draw the usual conclusions regarding the technology, which is possible when using a parametric functional form. (ii) DEA employs LP instead of regression analysis. Whereas a basic course in econometrics centered on the classical linear model is an essential ingredient of virtually every graduate program in economics, familiarity with LP can by no means be taken for granted. In textbook economics, constraints in standard optimization problems are typically assumed to be binding and Lagrange multipliers are almost always positive. (iii) This component is the most important of the three as it is non-statistical in nature and because the LP solution of a DEA problem produces no standard errors and leaves no room for hypothesis testing. But today DEA is a popular tool along with the other tools for relative efficiency estimation of DMUs. In some studies decision support tools were developed to allow 
DMU decision makers to change inputs and outputs attributes in order to see the change impact of the DMU efficiency (Alsmadi et al., 2015). DEA computes the relative efficiency of each DMU individually through the use of weighted averages and extension the Multiple Criteria of Decision Making (MCDM) models obtained CSW criterions for ranking specially when the number of peer DMUs is big (Smriti \& Khan, 2018). A CSW means only one production frontier hyperplane generates a compromised solution, all DMUs lie beneath the hyperplane and agree with the final status. Common weights, derived by Multi-Objective Linear Programming (MOLP), are theoretically supported by the concept of Pareto efficiency. Recently Yang et al., (2010) proposed a new approach to determine a bundle of CSW in DEA efficiency evaluation model by introducing a Multi-Objective Integer Programming (MOIP) and can be proven the solution is a Pareto efficient solution also. DEA and MOLP both search for set non-inferior solutions thus characterizing the DEA model by multi-objective programming is natural reasonable and appropriate. The CSW also can be used for ranking all DMUs. Hence, CSW idea can solve these two problems of classic DEA methods: finding common weights and ranking the peer DMUs. This idea was initially introduced by Cook, Roll, \& Kazakov (1990) and developed by Roll, Cook, \& Golany, (1991). Sinuany-Stern \& Friedman, (1998) extended DEA to provide the common weights in order to rank all the units on the same scale for given inputs and output sets. Liu \& Peng, (1999) introduced a model for obtaining the common weights, this model is a linear programming problem and presented a system to rank the DMUs. Also, they used this method for surveying group efficiency. Li \& Reeves, (1999) applied a multi-objective model that considers two additional efficiency measures: minimizing the sum of the DMU distances of frontier production (minisum) and minimizing the sum of the largest distance (minimax) from it, in addition to maximizing classical efficiency in DEA. Jahanshahloo et al., (2005) determined the common weights by the max-min method, then used the common weights for ranking efficient DMUs. Amin \& Toloo, (2007) proposed a CSW integrated DEA model to obtain the most efficient DMUs. Liu \& Peng, (2008) tried to introduce a methodology to determine one CSW for the performance indices of only DEA efficient DMUs. The ranking was done according to the efficiency score weighted by the common set of weights but Tarkhorani et al., (2014) showed that the criteria used by Liu et al. are not theoretically strong enough to discriminate when efficiency of DMUs are equal hence, their proposal is not generally correct and cannot provide an optimal solution. Agrell \& Bogetoft, (2010) introduced a game-theoretical approach to determine a set of CSWs in a setting where the DMUs must agree upon a common endogenous evaluation. In a recent study, Saati et al., (2012) have shown a two-phase CSW approach using an ideal virtual unit that is computationally efficient. Their model was applied in energy regulation using panel data from Danish district heating plants and Omrani, (2013) incorporated the uncertainty into the Zohrehbandian et al., (2010) common weight DEA model.

The object of the most of CSW techniques is defined on the best distance between a common weight with the other individual efficiency weights related to the inputs (outputs) position and "best distance" is defined a "minimized distance" usually. For example in an assumed CCR-Input Oriented, if for each input (output), $n$ weights are derived by $n$ repetitions on an optimization model (as will be described in $(I)$ ), in a special case, probably, the produced variance of $n$ input (output) weights can be big and this variance has the principal effect on the results of a CSW technique, during minimizing the distances. In these cases, minimizing cannot be useful. This research is tried to define another concept of the best distance and that is the closest distance to the importance individual efficiency weights related to the inputs (outputs). What is the concept of importance weights? The importance weights are the transformed weights by SVD on the two matrices (with $\mathrm{n} \times \mathrm{m}$ and $\mathrm{n} \times \mathrm{s}$ dimensions) defined by the values of the input and output efficient weights. After applying SVD, the variation between the other corresponding input (output) weights will be gathered around these (importance) weights.

The normalized eigenvalues which are produced by SVD will be named as degree of importance of the weights.

Now a hypothetical DMU ( $h$ DMU) can be defined such that each input (output) for $h$ DMU is found by a linear combination of the other corresponding $n$ inputs (outputs) and the coefficients in each combination would be the degree of importance of the weights. $h \mathrm{DMU}$ is a pivotal concept 
in the present research because the importance weights have bigger eigenvalues with respect to the others. Now if a CCR-Input Oriented case is applied for $h \mathrm{DMU}$ in a set of $n+1$ DMUs as will be illustrated in $(I V)$ the result would be a CSW such that the common weights are the closest weights to the importance individual efficiency weights related to the inputs (outputs). In section 2 we will formulate this method and the related CSW model, also an example will be illustrated in section 3 .

SVD can be introduced by correspondence analysis, a famous multivariate analysis in statistics, Guttman, (1959), Torgerson, (1958) and Hill, (1973) introduced correspondence analysis as a method of scaling rather than of contingency table analysis. It is convenient first to consider "gradient analysis", a simpler method of scaling which was developed by Whittaker, (1967) in the United States, Correspondence analysis can be regarded as a generalization of gradient analysis using the method of successive approximation. In general, SVD can be looked at from three mutually compatible points of view. On the one hand, we can see it as a method for transforming correlated variables into a set of uncorrelated ones that better expose the various relationships among the original data items. At the same time, SVD is a method for identifying and ordering the dimensions along which data points exhibit the most variation. This ties into the third way of viewing SVD, which is that once we have identified where the most variation is, it's possible to find the best approximation of the original data points using fewer dimensions. According to SVD logic, The Chi square-distance is a special case of a weighted Euclidean distance where the weights are the inverses of the corresponding average profile values. In principal component analysis (PCA), a method closely related to SVD, the $p$ dimensions are defined by continuous variables, often on different measurement scales. It is necessary to remove the effect of scale in some way, and this is usually done by dividing the data by the standard deviations of the respective variables. In this case the best-fitting line in each analysis is called a principal axis. More specifically it is referred to as the "first principal axis", since there are other principal axes, in SVD the inertia amount is accounted for by a principal axis is called a principal inertia, the first principal inertia in SVD refers to the first principal axis. It is also often called an eigenvalue because of the way it can be calculated, as an eigenvalue of a square symmetric matrix. Hence, SVD can be seen as a method for CSW to find the best weights for inhomogeneous inputs and outputs between $n$ observations (DMUs) simultaneously. Reduced SVD underlying the use of SVD for these tasks is that it takes the original data. Here this method consisting of some variant of the weights of input and output matrices which are found by CCR method and for each DMU in the first run (thus, for $m$ inputs and $s$ outputs we have one input weight matrix with dimension $n \times m$ and one output weight matrix with dimension $n \times s$ ) and breaks the matrices down into linearly independent components. These components are in some sense an abstraction away from the noisy correlations found in the inputs or outputs to sets of values that best approximate the underlying structure of the dataset along each dimension: $m$ or $s$ independently. Because the some of those components are very small, they can be ignored, resulting in an approximation of the data that contains substantially fewer dimensions less than $m$ and $s$. Reduce data dimensions to a few dimensions, described as $70 \%$ to $90 \%$ of the total variance in the data, generally.

\section{2- Model}

Let us assume that there are $n$ decision-making units (DMUs) each producing $s(n>s)$ different outputs from $m(n>m)$ different inputs. $X_{i j}$ is the input $i t h$ of unit $j t h$, while $Y_{r j}$ is the output $r t h$ of unit $j t h$ and $X_{i j}$ 's and $Y_{r j}$ 's are positive. For determining a CSW for DMUs according to Cook et al., (1990), Jahanshahloo et al., (2005), Roll et al., (1991) we need to find the weights in two runs: in the first run the efficiency of the individual DMUs will be found and in the second run, CSW will be found by optimization on the whole of individual efficiencies in one step. Here some activities will be added in the first run and that is finding the degree of importance of the weights which is derived by applying SVD such that some of DMUs that have the main influence in a combination of input (output) weights with respect to the others, will be appeared this fact can be seen by finding the normalized eigenvalues of input (output) weight matrices as will described in $(I)$. Now a hypothetical DMU ( $h$ DMU) with a set of spectral inputs and outputs can be introduced and added 
to the set of observations (DMUs). Essentially spectral inputs (outputs) are a linear combination of main inputs (outputs) such that the coefficients of these linear models have been found by degree of importance (normalized eigenvalues ) of the inputs (outputs) weight matrix. Finally, in the second run, a CCR multiplier model can be applied for hDMU among peer $n+1$ DMUs. It would be clear the result of solving the model can be a CSW because hDMU has been contained in all information about the input and output weights of all $\mathrm{n}$ DMUs. Also the result would be a whole common set of weights and the common weights will be determined with multiplication this quantity by the degree of importance of each DMU from run (I). On the contrary other criterions, it is clear that in the present criterion the specific common weight for each input (output) in each DMU can differ with the other DUMs. One of the most important benefits of this criterion is dimensionally reduction such that for calculation of CSW, similar DMUs with similar relation between input and output weights have a pale influence for the others and can be reduced but the dissimilar relation between input and output weights have been included with strong influence.

In the following, the model has been described in details accompany with CCR multiplier model. Suppose in the first run two input and output weight matrices have found $\left(\boldsymbol{V}_{n m}, \boldsymbol{U}_{n s}\right)$ by CCR method as follows:

$1^{\text {st }}$ Run:

$$
\begin{gathered}
\max _{p} \sum_{r=1}^{s} u_{r}^{p} y_{r p}, \quad p=1, \ldots, n \\
s t: \sum_{i=1}^{m} v_{i}^{p} x_{i p}=1 \\
\sum_{r=1}^{s} u_{r}^{p} y_{r j}-\sum_{i=1}^{m} v_{i}^{p} x_{r j} \leq 0, j=1, \ldots, n \rightarrow U_{n s}=\left[u_{r}^{p}\right]_{n \times s} \\
u_{r}^{p}, v_{i}^{p} \geq 0, \quad i=1, \ldots, m, r=1, \ldots, s
\end{gathered}
$$

Let $\boldsymbol{V}_{n m}$ and $\boldsymbol{U}_{n s}$ be nonsingular then by SVD method we write:

$$
\left.\begin{array}{clc}
\boldsymbol{V}_{n m}=\boldsymbol{G}_{n m}^{V} \boldsymbol{D}_{m m}^{V} \boldsymbol{A}_{m m}^{(V) T}, & \boldsymbol{A}_{m m}^{V}=\left[a_{t l}^{V}\right]_{t, l=1, \ldots, m}, & \boldsymbol{D}_{m m}^{V}=\left[d_{t l}^{V}\right]_{t, l=1, \ldots, m} \\
\boldsymbol{U}_{n s}=\boldsymbol{G}_{n s}^{U} \boldsymbol{D}_{s s}^{U} \boldsymbol{A}_{s s}^{(U) T}, & \boldsymbol{A}_{s s}^{U}=\left[a_{f q}^{U}\right]_{f, q=1, \ldots, s}, & \boldsymbol{D}_{s s}^{U}=\left[d_{f q}^{U}\right]_{f, q=1, \ldots, s}
\end{array}\right\}
$$

Where $\boldsymbol{M}^{T} \equiv$ Transpose matrix of $\boldsymbol{M}$ hence $\boldsymbol{G}^{(V) T} \boldsymbol{G}^{V}=\boldsymbol{I}_{m m}, \quad \boldsymbol{G}^{(U) T} \boldsymbol{G}^{U}=\boldsymbol{I}_{s s}$, where $\boldsymbol{I}=\left[\begin{array}{c}1 \ldots \\ \ldots \ldots \\ 0 \ldots 1\end{array}\right]$ the columns of $\boldsymbol{G}^{V}$ and $\boldsymbol{G}^{U}$ are orthonormal eigenvectors of $\boldsymbol{V} \boldsymbol{V}^{T}$ and $\boldsymbol{U} \boldsymbol{U}^{T}$ respectively, also the columns of $\boldsymbol{A}^{V}$ and $\boldsymbol{A}^{U}$ are orthonormal eigenvectors of $\boldsymbol{V}^{T} \boldsymbol{V}$ and $\boldsymbol{U}^{T} \boldsymbol{U}$, $\boldsymbol{D}^{V}$ and $\boldsymbol{D}^{U}$ are diagonal matrices containing the square roots of eigenvalues from $\boldsymbol{G}^{V}$ or $\boldsymbol{A}^{V}$ and $\boldsymbol{G}^{U}$ or $\boldsymbol{A}^{U}$ in descending order respectively. Now define vectors $\boldsymbol{B}^{V}$ and $\boldsymbol{B}^{U}$ (the weight vectors of the eigenvalues of $\boldsymbol{G}^{V}$ (or $\boldsymbol{A}^{V}$ ) and $\boldsymbol{G}^{U}$ (or $\boldsymbol{A}^{U}$ ) matrices, respectively) as follows:

$$
\left.\begin{array}{ll}
d_{t .}^{V}=\sum_{l=1}^{m} d_{t l}^{V}, \quad b_{t}^{V}=\frac{d_{t .}^{(V) 2}}{\sum_{t=1}^{m} d_{t .}^{(V) 2}}, \quad \boldsymbol{B}_{m 1}^{V}=\left[b_{t}^{V}\right]_{t=1, \ldots, m} \\
d_{f .}^{U}=\sum_{q=1}^{s} d_{f q}^{U}, \quad b_{f}^{U}=\frac{d_{f .}^{(U) 2}}{\sum_{f=1}^{s} d_{f .}^{(U) 2}}, \quad \boldsymbol{B}_{s 1}^{U}=\left[b_{f}^{U}\right]_{f=1, \ldots, s}
\end{array}\right\}
$$

We can see that the elements of $\boldsymbol{B}^{V}$ and $\boldsymbol{B}^{U}$ are normalized eigenvalues which are found for $\boldsymbol{V}$ and $\boldsymbol{U}$ respectively and some of these elements which are very small can be ignored. The eliminated elements can be identified by eigenvalues and reduction dimension of vectors: $\boldsymbol{B}^{V}$ and 
$\boldsymbol{B}^{U}$ and also matrices: $\boldsymbol{A}^{V}$ and $\boldsymbol{A}^{U}$. As described in the above, the elements of the vectors $\boldsymbol{B}^{V}$ and $\boldsymbol{B}^{U}$ are the normalized principal inertias (eigenvalues) and according to the concept of inertia which is described at section 1 the variances are also equal to the inertias of the projected inputs (outputs) profiles therefore these elements should describe the portion of variance for the input and output weights respectively. Let $m_{0}$ be the reduced dimension of inputs $\left(m_{0}<m\right.$ ) and $s_{0}$ be the reduced dimension of outputs $\left(s_{0}<s\right)$ when an accepted percent (instead of 100 percent) of the variations has been selected therefore we can define the estimation of matrices $V$ and $\boldsymbol{U}$ as $\widehat{\boldsymbol{V}}$ and $\widehat{\boldsymbol{U}}$ according to the following conditions:

$$
\begin{aligned}
& \sum_{t=1}^{m_{0}} b_{t}^{V} \cong 0.9, \widehat{\boldsymbol{V}}_{n m_{0}}=\boldsymbol{G}_{n m_{0}}^{V} \boldsymbol{D}_{m_{0} m_{0}}^{V} \boldsymbol{A}_{m_{0} m_{0}}^{(V) T} \\
& \sum_{f=1}^{s_{0}} b_{f}^{U} \cong 0.9, \quad \widehat{\boldsymbol{U}}_{n s_{0}}=\boldsymbol{G}_{n s_{0}}^{U} \boldsymbol{D}_{s_{0} s_{0}}^{U} \boldsymbol{A}_{s_{0} s_{0}}^{(U) T}
\end{aligned}
$$

If we let:

Now let: $\Delta_{n \times 1}=\boldsymbol{G}_{n m_{0}}^{V} \boldsymbol{B}_{m_{0} 1}^{V}$ and $\boldsymbol{\Omega}_{n \times 1}=\boldsymbol{G}_{n s_{0}}^{U} \boldsymbol{B}_{s_{0} 1}^{U}$, after some simplification we have: $\Delta_{n \times 1}=\boldsymbol{V}_{n m_{0}} \boldsymbol{A}_{m_{0} m_{0}}^{V} \boldsymbol{D}_{m_{0} m_{0}}^{(V)-1} \boldsymbol{B}_{m_{0} 1}^{V}$ or if $\tau=\sum_{t=1}^{m_{0}} d_{t}^{(V) 2}$

then $\Delta_{n \times 1}=\frac{1}{\tau} \boldsymbol{V}_{n m_{0}} \boldsymbol{A}_{m_{0} m_{0}}^{V} \mathbf{1}_{m_{0} 1}$ where $\mathbf{1}_{m_{0} 1}=\left[\begin{array}{c}1 \\ \cdot \\ \cdot \\ 1\end{array}\right]_{m_{0} \times 1} \quad$ and by the same reason,

if $\eta=\sum_{f=1}^{s_{0}} d_{f}^{(U) 2}$ then $\boldsymbol{\Omega}_{n \times 1}=\frac{1}{\eta} \boldsymbol{U}_{n s_{0}} \boldsymbol{A}_{s_{0} s_{0}}^{U} \mathbf{1}_{s_{0} 1}$.

Now assume that we have one hypothetical DMU with $m$ input and $s$ output such that each element of $\Delta_{n \times 1}=\left[\delta_{j}\right]_{j=1, \ldots, n}$ is the weight of the $m$ input efficiency coefficients for a linear combination of $n$ main DMUs inputs and each element of $\boldsymbol{\Omega}_{n \times 1}=\left[\omega_{j}\right]_{j=1, \ldots, n}$ is the weight of $s$ output efficiency coefficients for a linear combination of $n$ main DMUs outputs. Consequently the Common Weights of efficiency coefficients for all of $n$ DMUs like $\alpha$ and $\beta$ can be found by the following optimization:

$2^{\text {nd }}$ Run:

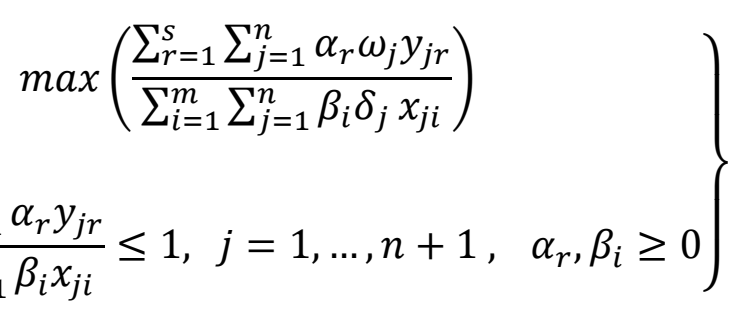

For understanding the difference between using this model with respect to the other popular methods when the variation of the weights is high, this method has been compared Global criterion thus, in the following section by a numerical example. We know that the optimization of Global criterion can be defined in two runs according to the following programming:

Run1:

Run2:

$\left\{\begin{array}{c|c}\min \theta_{p}, \quad \forall p=1, \ldots, n \\ \text { st. } \sum_{j=1}^{n} \lambda_{j} x_{i j} \leq \theta_{p} x_{i p}, \quad i=1, \ldots, m \\ \sum_{j=1}^{n} \lambda_{j} y_{r j} \leq y_{r p}, j=1, \ldots, s & \min \sum_{p=1}^{n}\left[\left(\theta_{p}-\frac{\sum_{r=1}^{s} \alpha_{r j}^{g} y_{r j}}{\sum_{i=1}^{m} \beta_{i j}^{g} x_{i j}}\right)\right], \theta_{p} \text { calculated from Run } 1 \\ & \text { st. } \frac{\sum_{r=1}^{s} \alpha_{r j}^{g} y_{r j}}{\sum_{i=1}^{m} \beta_{i j}^{g} x_{i j}} \leq 1, \alpha_{r j}^{g}, \beta_{i j}^{g} \geq 0, j=1, \ldots, n\end{array}\right\}$ 


\section{3- Numerical Example}

For illustrating the potential of the model, we will review the numerical illustration was first analyzed in (Hokkanen \& Salminen, 1997) in which 22 solid waste management treatment systems in the Oulu region of Finland have compared over 5 inputs and 3 outputs (defined in Sarkis, 2000). The original data is presented in the following Table:

Table1: Information of 22 solid waste management treatment systems in the Oulu region of Finland with 5 inputs and 3 outputs (see Sarkis, 2000)

\begin{tabular}{|c|c|c|c|c|c|c|c|c|}
\hline \multicolumn{3}{|c|}{ Outputs } & \multicolumn{5}{|c|}{ Inputs } & \multirow[t]{2}{*}{$\overline{\text { DMU }}$} \\
\hline $\begin{array}{l}\text { Resource } \\
\text { recovery }\end{array}$ & Employees & $\begin{array}{l}\text { Technical } \\
\text { feasibility }\end{array}$ & $\begin{array}{c}\text { Surface } \\
\text { water } \\
\text { releases }\end{array}$ & $\begin{array}{c}\text { Acidification } \\
\text { releases }\end{array}$ & $\begin{array}{l}\text { Health } \\
\text { Effects }\end{array}$ & $\begin{array}{l}\text { Global } \\
\text { Effects }\end{array}$ & Cost & \\
\hline 13,900 & 14 & 5.00 & 670 & 1190 & 609 & $552,678,100$ & 656 & 1 \\
\hline 23,600 & 18 & 4.00 & 682 & 1190 & 575 & $539,113,200$ & 786 & 2 \\
\hline 39,767 & 24 & 4.00 & 594 & 1222 & 670 & $480,565,400$ & 912 & 3 \\
\hline 13,900 & 10 & 9.00 & 443 & 1191 & 411 & $559,780,715$ & 589 & 4 \\
\hline 23,600 & 14 & 7.00 & 404 & 1191 & 325 & $532,286,214$ & 706 & 5 \\
\hline 40,667 & 18 & 6.50 & 384 & 1226 & 500 & $470,613,514$ & 834 & 6 \\
\hline 13,900 & 10 & 9.00 & 420 & 1191 & 398 & $560,987,877$ & 580 & 7 \\
\hline 23,600 & 14 & 7.00 & 393 & 1191 & 314 & $532,224,858$ & 682 & 8 \\
\hline 41,747 & 22 & 6.50 & 373 & 1229 & 501 & $466,586,058$ & 838 & 9 \\
\hline 13,900 & 9 & 9.00 & 405 & 1191 & 373 & $561,555,877$ & 579 & 10 \\
\hline 23,600 & 13 & 7.00 & 370 & 1191 & 292 & $532,302,258$ & 688 & 11 \\
\hline 42,467 & 17 & 6.50 & 361 & 1230 & 499 & $465,356,158$ & 838 & 12 \\
\hline 13,900 & 12 & 9.00 & 538 & 1191 & 500 & $560,500,215$ & 595 & 13 \\
\hline 23,600 & 17 & 7.00 & 489 & 1191 & 402 & $532,974,014$ & 709 & 14 \\
\hline 40,667 & 20 & 6.50 & 538 & 1226 & 648 & $474,137,314$ & 849 & 15 \\
\hline 13,900 & 12 & 9.00 & 538 & 1191 & 500 & $560,500,215$ & 604 & 16 \\
\hline 23,600 & 17 & 7.00 & 489 & 1191 & 402 & $532,974,014$ & 736 & 17 \\
\hline 40,667 & 20 & 6.50 & 538 & 1226 & 648 & $474,137,314$ & 871 & 18 \\
\hline 13,900 & 7 & 9.00 & 558 & 1193 & 495 & $568,674,539$ & 579 & 19 \\
\hline 23,600 & 18 & 6.00 & 535 & 1195 & 424 & $536,936,873$ & 695 & 20 \\
\hline 45,167 & 16 & 7.00 & 513 & 1237 & 651 & $457,184,239$ & 827 & 21 \\
\hline 45,167 & 16 & 7.00 & 513 & 1239 & 651 & $457,206,173$ & 982 & 22 \\
\hline
\end{tabular}

By optimization (I) and continuing with model (II) (Details of output by SAS in Appendix) we have 3 dimension reduction for the inputs because as illustrated in Diagonal of $\boldsymbol{D}_{5,5}^{V}$ matrix which is located in the Appendix. The first two elements of this matrix have more than 85 percent (accepted percent) of inputs information and by this percent we can have 2 dimension reductions for the outputs and by Diagonal of $\boldsymbol{D}_{3,3}^{U}$, it can be seen, more than 72 percent (accepted percent) of information is in the first eigenvalue. Therefore 2 dimension reductions will be considered hence $m_{0}=2$ and $s_{0}=1$. If the optimization is continued by (III) and according to $\boldsymbol{\Delta}_{22,1}$ and $\boldsymbol{\Omega}_{22,1}$ and by $(I V)$ the weights can be derived. The following values show the comparative results between 
SVD method and Global criterion, solved by $(I V)$ and $(V)$ :

Table2: Comparison of calculated CSW by SVD and Global criterion

\begin{tabular}{rrrr}
\hline \multicolumn{1}{c}{$\boldsymbol{\alpha}$} & $\boldsymbol{\beta}$ & \multicolumn{1}{c}{$\boldsymbol{\alpha}^{\boldsymbol{g}}$} & $\boldsymbol{\beta}^{\boldsymbol{g}}$ \\
\hline $\mathbf{1 . 0 2 5 5}$ & 0.050969483 & 12.2867450 & 0.226332 \\
$\mathbf{2 . 4 4 8 8}$ & 0.368120608 & 2.5031091 & 0.000000 \\
$\mathbf{3 7 2 6 . 1 4 8 3}$ & 0.000044326 & 0.0022605 & 0.030266 \\
& 0.003909605 & & 0.019912 \\
& 0.003167122 & & 0.000000 \\
\hline
\end{tabular}

It must be noted, $\boldsymbol{\alpha}$ and $\boldsymbol{\beta}$ are the whole common weights but CSW for each DMU will be derived by multiplication of $\boldsymbol{\alpha}$ (or $\boldsymbol{\beta}$ ) in $\boldsymbol{\Delta}_{22,1}$ (or $\boldsymbol{\Omega}_{22,1}$ ) which are located at the Appendix respectively (degrees of importance) for example, CSW for $\mathrm{DMU}_{3}, \mathrm{DMU}_{20}$ and $\mathrm{DMU}_{21}$, is illustrated in table3.

\begin{tabular}{|c|c|c|c|c|c|c|c|c|}
\hline$\alpha \omega_{D M U}$ & $\beta \boldsymbol{\delta}_{D M U}$ & DMU & $\alpha \omega_{D M U}$ & $\beta \delta_{D M U}$ & DMU & $\alpha \omega_{D M U}$ & $\beta \delta_{D M U}$ & DMU \\
\hline 0 & 0.000294 & \multirow{5}{*}{21} & 0.0005 & 0.005772 & \multirow{5}{*}{20} & 0.00126 & 0.004137 & \multirow{5}{*}{3} \\
\hline 0 & 0.002124 & & 0.0012 & 0.041689 & & 0.0030098 & 0.030061428 & \\
\hline \multirow[t]{3}{*}{0000005} & 0.0000002 & & 1.8499 & 0.000005 & & 4.5798089 & 0.0000036 & \\
\hline & 0.00002 & & & 0.000443 & & & 0.000319266 & \\
\hline & 0.000018 & & & 0.000359 & & & 0.000258633 & \\
\hline
\end{tabular}

Table3: CSW of 3 selected DMUs, calculated by table2 and degrees of importance according to $\boldsymbol{\Delta}_{22,1}$ and $\boldsymbol{\Omega}_{22,1}$

\section{4-Results}

The main difference between this criterion with the others is the difference between the common weights for each DMU, because in this method first a whole weight is derived and this quantity will be multiplied by each individual degree of importance which is found in the first run. The other result can be the differences between input efficient weights among output efficient weights which will be derived from the observations (DMUs). For example according to matrix $V_{22 \times 5}($ column=2) which is located in the Appendix, the efficiency weights of "Global Effects" are zero for all of 22 DMUs and for this reason $\beta^{g}=0$ but by SVD method, the biggest whole weight is for this input (i.e. $\beta=0.368120608$ ). Also by global criterion, the efficient common weight of the output: "Resource Recovery" is $\alpha^{g}=0.0022605$ and by SVD, the whole weight is $\alpha$ $=3726.1483$, how these variations can be explained? $\alpha_{\text {Resource Recovery }}$ is very high, because, not only the coefficient of variation of the weights of Resource Recovery" is very high, (it is calculated 1.477 or 147.7 percent) but also the coefficient of variation of this output is high with respect to the others, therefore a big common weight for this output is not unexpected by SVD method.

The Coefficients of Variation (CV) of 3 outputs and 5 inputs in the above example are compared as follows:

$$
\begin{aligned}
& \mathrm{CV}_{\text {Technical_Feasibility }}=0.215815, \quad \mathrm{CV}_{\text {Employees }}=0.281607, \quad \mathrm{CV}_{\text {Resource_Recovery }}=0.447905 \\
& \mathrm{CV}_{\text {Cost }}=0.165932, \quad \mathrm{CV}_{\text {Global_Effects }}=0.78758296, \quad \mathrm{CV}_{\text {Health }} \quad \text { Effects }=0.244442 \text {, }
\end{aligned}
$$

$\mathrm{CV}_{\text {Acidification_Releases }}=0.015834, \mathrm{CV}_{\text {Surface_water_Releases }}=0.191632$.

In the first of view, it is clear that variation of Resource Recovery" and "Global Effects" are high.

In the above example, $h \mathrm{DMU}$ can be the $23^{\text {th }}$ observation (DMU) with spectral inputs (outputs) that are linear combinations of the other DMUs' inputs (outputs), the linear input coefficient set 
are listed in the $\boldsymbol{\Delta}_{22,1}$ and the linear output coefficient set are listed in the $\Omega_{22,1}$. According to these vectors we have:

Table4: Derived information of $h$ DMU

\begin{tabular}{|c|c|c|c|c|c|c|c|c|}
\hline \multicolumn{3}{|c|}{ Outputs } & \multicolumn{5}{|c|}{ Inputs } & DMU \\
\hline Resource & Employees & $\begin{array}{l}\text { Technical } \\
\text { feasibility }\end{array}$ & $\begin{array}{l}\text { Surface } \\
\text { water } \\
\text { releases }\end{array}$ & $\begin{array}{c}\text { Acidification } \\
\text { releases }\end{array}$ & $\begin{array}{l}\text { Health } \\
\text { Effects }\end{array}$ & $\begin{array}{l}\text { Global } \\
\text { Effects }\end{array}$ & Cost & $h \mathrm{DMU}$ \\
\hline 109,121 & 65 & 30.30 & 1443 & 3812 & 1366 & $1,704,610,000$ & 2142 & 23 \\
\hline
\end{tabular}

If coefficient of variation of inputs (outputs) are calculated for 23 DMUs we will have the following changes:

$\mathrm{CV}_{\text {Technical_Feasibility }}=0.631566597, \quad \mathrm{CV}_{\text {Employees }}=0.638053649, \quad \mathrm{CV}_{\text {Resource_Recovery }}=0.676458116$ $\mathrm{CV}_{\text {Cost }}=0.398746406, \quad \mathrm{CV}_{\text {Global_Effects }}=0.439340704, \quad \mathrm{CV}_{\text {Health Effects }}=0.410481699$, $\mathrm{CV}_{\text {Acidification_Releases }}=0.412512120, \mathrm{CV}_{\text {Surface_water_Releases }}=0.413231790$.

It can be observed the biggest output $\mathrm{CV}$ is for resource recovery and the biggest input $\mathrm{CV}$ is for global_effects also the differences between CVs for inputs and outputs are less than the first case (22 DMUs) .

\section{5-Conclusions}

As pointed before, SVD method can be useful when there are uncoordinated changes between outputs or inputs and also between the eigenvalues of matrices $\boldsymbol{V}$ or $\boldsymbol{U}$ which are defined in section2. In addition when SVD is applied we can classify the results by the following sentences briefly:

1- $\quad$ CSW for each DMU will be defined and found by multiplication of two values:

- The whole CSW which is found by $(I V)$.

- Degree of importance of each DMU (i.e. $\boldsymbol{\Delta}_{22,1}$ and $\boldsymbol{\Omega}_{22,1}$ )

2- $\quad$ For $n$ observations when the variation of an output (input) with respect to the others is high, we can expect the common weight of that output (input) will be bigger than the others.

3- $\quad$ For $n$ observations, if the variation of corresponding individual relative output (input) efficient weights with respect to the others is high then we can expect the similar common weight is high also if both items 1 and 2 occur simultaneously and the weights will be increasingly exacerbated.

4- $\quad$ Definition of $h \mathrm{DMU}$ as defined before, can close the changes between the outputs (inputs) for peer $n+1$ DMUs, on the other hand inputs (outputs) of $h \mathrm{DMU}$ have the whole information of inputs (outputs) of the other DMUs therefore, solving a CCR model on $h$ DMU like (IV) can give us a whole CSW. 


\section{6-Refferences}

[1] Agrell, P. J., \& Bogetoft, P. (2010). Endogenous generalized weights under DEA control.

[2] Alsmadi, I., Najadat, H., \& Al-oqily, I. (2015). Evaluating the importance of the weight factors in DEA optimisation. Journal of Data Science, 1((2)). http://doi.org/JDS. 1.

10.1504/IJDS.2015.072410.

[3] Amin, G. R., \& Toloo, M. (2007). Finding the most efficient DMUs in DEA: An improved integrated model. Computers \& Industrial Engineering, 52(1), 71-77. Computers \& Industrial Engineering, 52(1), 71-77.

[4] Charnes, A., Cooper, W. W., \& Rhodes, E. (1978). Measuring the efficiency of Decision Making Units. European Journal of Operational Research, 2, 429-444.

[5] Cook, W. D., Roll, Y., \& Kazakov, A. (1990). A DEA model for measuring the relative efficiencies of highway maintenance patrols. INFOR, 28(2), 113-124.

[6] Hokkanen, J., \& Salminen, P. (1997). Electre III and IV methods in an environmental problem. Journal of Multi-Criteria Analysis, 6, 216-226.

[7] Jahanshahloo, G. R., Memariani, A., Hosseinzadeh Lotfi, F., \& Rezaei, H. Z. (2005). A note on some DEA models and finding efficiency and complete ranking using common set of weights. Applied Mathematics and Computation, 166, 265-281.

[8] Li, X. B., \& Reeves, G. R. (1999). A multiple criteria approach to data envelopment analysis. European Journal of Operations Research, 115, 507-517.

[9] Liu, F. H. F., \& Peng, H. (1999). Ranking of units on the DEA frontier with common weights. Computers and Operational Research, 35, 1624-1637.

[10] Liu, F. H. F., \& Peng, H. . (2008). Ranking of units on the DEA frontier with common weights. Computers \& Operations Research, 35(5), 1624-1637.

[11] Omrani, H. (2013). Common weights data envelopment analysis with uncertain data: A robust optimization approach. Computers \& Industrial Engineering, 66, 1163-1170.

[12] Roll, Y., Cook, W. D., \& Golany, B. (1991). Controlling factor weights in data envelopment analysis. IIE Transactions, 23, 2-9.

[13] Saati, S., Hatami-Marbini, A., Agrell, P. J., \& Tavana, M. (2012). A common set of weight approach using an ideal decision making unit in data envelopmentanalysis. Journal of Industrial and Management Optimization, 8(3), 623-637.

[14] Sarkis, J. (2000). A comparative analysis of DEA as a discrete alternative multiple criteria decision tool, 123(European J Oprl Res), 543-557.

[15] Sinuany-Stern, Z., \& Friedman, L. (1998). DEA and discriminate analysis of ratios for ranking units. European Journal of Operational Research, 111, 470-478.

[16] Smriti, T. N., \& Khan, H. R. (2018). Efficiency Analysis of Manufacturing Firms Using Data Envelopment Analysis Technique, 18, 69-78.

[17] Subhash Ray, C. (2004). Data Envelopment Analysis: Theory and Techniques for Economic and Operations Research.

[18] Tarkhorani, S. ., Khodabakhshi, M., Mehrabanian, S., \& Nouri-Bahmani, F. (2014). Ranking 
decision-making units using common weights in DEA. Applied Mathematical Modelling, 38(15-16), 3890-3896.

[19] Yang, F., Yang, C., \& Liang, L. (2010). New approach to determine common weights in DEA efficiency evaluation model. Journal of Systems Engineering and Electronics, 21(4), 609-615.

[20] Zohrehbandian, M., Makui, A., \& Alinezhad, A. (2010). A compromise solution approach for finding common weights in DEA: An improvement to Kao and Hung's approach. Journal of the Operational Research Society, 61, 604-610.

\section{7-Appendix : SAS output}

$$
V_{22 \times 5}=\left[\begin{array}{ccccc}
0.0015244 & 0.000000 & 0.000000 & 0.000000 & 0.000000 \\
0.0012723 & 0.000000 & 0.000000 & 0.000000 & 0.000000 \\
0.0010965 & 0.000000 & 0.000000 & 0.000000 & 0.000000 \\
0.000000 & 0.000000 & 0.000000 & 0.000000 & 0.000000 \\
0.000000 & 0.000000 & 0.000804 & 0.000620 & 0.000000 \\
0.000000 & 0.000000 & 0.000419 & 0.000645 & 0.000192 \\
0.0016819 & 0.000000 & 0.001187 & 0.000526 & 0.000584 \\
0.0009644 & 0.000000 & 0.000736 & 0.000000 & 0.000283 \\
0.000000 & 0.000000 & 0.000000 & 0.000814 & 0.000000 \\
0.0088217 & 0.000000 & 0.001312 & 0.000000 & 0.000000 \\
0.000000 & 0.000000 & 0.003427 & 0.000000 & 0.000512 \\
0.0011838 & 0.000000 & 0.000591 & 0.000000 & 0.000000 \\
0.000000 & 0.000000 & 0.000831 & 0.000559 & 0.000000 \\
0.000000 & 0.000000 & 0.000000 & 0.000816 & 0.000000 \\
0.000000 & 0.000000 & 0.000000 & 0.000117 & 0.000000 \\
0.000000 & 0.000000 & 0.001075 & 0.000477 & 0.000000 \\
0.000000 & 0.000000 & 0.000000 & 0.000816 & 0.000000 \\
0.000000 & 0.000000 & 0.000000 & 0.000000 & 0.000000 \\
0.0017271 & 0.000000 & 0.000000 & 0.000000 & 0.000000 \\
0.0007972 & 0.000000 & 0.001052 & 0.000000 & 0.000000 \\
0.000000 & 0.000000 & 0.000000 & 0.000808 & 0.000000 \\
0.000000 & 0.000000 & 0.000525 & 0.000000 & 0.000000
\end{array}\right]
$$


Hassan Naseri , S.Esmaeil Najafi ,Abbas Saghaei

429

\begin{tabular}{|c|c|c|}
\hline \multicolumn{3}{|c|}{$\boldsymbol{G}_{22,3}^{U}$} \\
\hline 0.1572948 & 0.2748323 & -0.019356 \\
\hline 0.041127 & 0.3780334 & 0.0522484 \\
\hline 0.0332139 & 0.3284327 & 0.0465259 \\
\hline 0 & 0 & 0 \\
\hline 0.2868582 & 0.0007231 & -0.085278 \\
\hline 0.2605745 & -0.167783 & 0.113525 \\
\hline 0.224767 & -0.0199 & 0.2884307 \\
\hline 0.22496 & 0.1761803 & -0.068278 \\
\hline 0.2688359 & -0.107306 & -0.009981 \\
\hline 0.2603521 & 0.104047 & -0.09771 \\
\hline 0.0600574 & 0.593872 & 0.1278307 \\
\hline $3.8963 \mathrm{E}-9$ & $-1.107 \mathrm{E}-8$ & 0.6118369 \\
\hline 0.2092335 & 0.1845883 & -0.059626 \\
\hline 0.2716883 & 0.0178262 & -0.118951 \\
\hline 0.2694914 & -0.107576 & -0.010327 \\
\hline 0.3016574 & -0.121118 & -0.158448 \\
\hline 0.2249546 & 0.1105172 & -0.080566 \\
\hline 0.2694914 & -0.107576 & -0.010327 \\
\hline 0.2468578 & -0.170589 & 0.337687 \\
\hline 0.1341541 & 0.2839363 & -0.007236 \\
\hline $3.618 \mathrm{E}-9$ & $-1.028 \mathrm{E}-8$ & 0.5681343 \\
\hline 0.3067649 & -0.211987 & -0.002945 \\
\hline
\end{tabular}

\begin{tabular}{|c|c|c|c|c|c|}
\hline & \multicolumn{3}{|c|}{$\boldsymbol{A}_{3,3}^{U}$} & $\Delta_{22,1}$ & $\boldsymbol{\Omega}_{22,1}$ \\
\hline 0.1923714 & 0.9667888 & -0.255576 & -0.000067 & 0.1135297 & 0.005821 \\
\hline 0.0735911 & 0.2555764 & 0.9667888 & 0.0000426 & 0.0947546 & 0.001522 \\
\hline 0.0000229 & 0.0000535 & -0.000058 & 1 & 0.0816619 & 0.0012291 \\
\hline & & & & 0 & 0 \\
\hline & & & & 0.0456048 & 0.0106158 \\
\hline & & & & 0.0273743 & 0.0096431 \\
\hline & & & & 0.1937893 & 0.008318 \\
\hline & & & & 0.1114471 & 0.0083251 \\
\hline & & & & 0.0058133 & 0.0099488 \\
\hline & & & & 0.7241905 & 0.0096349 \\
\hline & & & & 0.1790042 & 0.0022225 \\
\hline & & & & 0.1184316 & $1.442 \mathrm{E}-10$ \\
\hline & & & & 0.0465519 & 0.0077431 \\
\hline & & & & 0.0058276 & 0.0100544 \\
\hline & & & & 0.0008356 & 0.0099731 \\
\hline & & & & 0.0584628 & 0.0111634 \\
\hline & & & & 0.0058276 & 0.0083249 \\
\hline & & & & 0 & 0.0099731 \\
\hline & & & & 0.1286258 & 0.0091355 \\
\hline & & & & 0.1132497 & 0.0049646 \\
\hline & & & & 0.0057705 & $1.48 \mathrm{E}-10$ \\
\hline & & & & 0.0268879 & 0.0113524 \\
\hline
\end{tabular}




\begin{tabular}{|c|c|c|c|c|c|c|c|c|c|c|}
\hline \multicolumn{5}{|c|}{$\boldsymbol{G}_{22,5}^{V}$} & $\begin{array}{l}\text { Diagonal } \\
\text { of } \boldsymbol{D}_{5,5}^{V}\end{array}$ & \multicolumn{5}{|c|}{$\boldsymbol{A}_{5,5}^{V}$} \\
\hline 0.1528774 & -0.075948 & 0.0154964 & 0.0318113 & -0.059085 & 0.0097532 & 0.978 & -0.207 & 0.02 & 0.01 & 0 \\
\hline 0.1275951 & -0.063388 & 0.0129336 & 0.0265505 & 0.1604281 & 0.0041494 & 0 & 0 & 0 & 0 & 1 \\
\hline 0.1099646 & -0.054629 & 0.0111465 & 0.0228818 & -0.48417 & 0.0019736 & 0.207 & 0.953 & -0.166 & -0.146 & 0 \\
\hline 0 & 0 & 0 & 0 & 0 & 0.0005676 & 0.0150 & 0.165 & 0.986 & -0.023 & 0 \\
\hline 0.0179863 & 0.2093829 & 0.2419042 & -0.231581 & 0.3152317 & 0 & 0.019 & 0.147 & -0.002 & 0.989 & 0 \\
\hline 0.010244 & 0.1287336 & 0.2866942 & 0.2007516 & -0.394415 & & & & & & \\
\hline 0.1957693 & 0.2304624 & 0.1792544 & 0.7267092 & 0.1123499 & & & & & & \\
\hline 0.1128616 & 0.1310139 & -0.0525 & 0.3244082 & -0.063817 & & & & & & \\
\hline 0.0012524 & 0.0324376 & 0.4066081 & -0.033193 & -0.187663 & & & & & & \\
\hline 0.9124952 & -0.138146 & -0.020957 & -0.152555 & 0.0135223 & & & & & & \\
\hline 0.0736 & 0.8052709 & -0.289416 & 0.0128347 & 0.0550301 & & & & & & \\
\hline 0.1312397 & 0.0767723 & -0.037802 & -0.126942 & 0.0398742 & & & & & & \\
\hline 0.0184644 & 0.2131539 & 0.2091568 & -0.236021 & 0.1136622 & & & & & & \\
\hline 0.0012555 & 0.0325173 & 0.4076071 & -0.033274 & 0.2199776 & & & & & & \\
\hline 0.00018 & 0.0046624 & 0.0584437 & -0.004771 & -0.033157 & & & & & & \\
\hline 0.0235073 & 0.2659322 & 0.147621 & -0.295286 & -0.541548 & & & & & & \\
\hline 0.0012555 & 0.0325173 & 0.4076071 & -0.033274 & 0.2199776 & & & & & & \\
\hline 0 & 0 & 0 & 0 & 0 & & & & & & \\
\hline 0.1732055 & -0.086046 & 0.0175569 & 0.0360413 & 0.0639817 & & & & & & \\
\hline 0.1022348 & 0.2019234 & -0.080606 & -0.253298 & 0.0155999 & & & & & & \\
\hline 0.0012432 & 0.0321985 & 0.403611 & -0.032948 & -0.009564 & & & & & & \\
\hline 0.0111219 & 0.1205907 & -0.044271 & -0.13471 & 0.1272904 & & & & & & \\
\hline
\end{tabular}

